\title{
Modelling of Carbon-Rich Stars with Far Infrared Flux Excess
}

\section{STEFANO BAGNULO ${ }^{1}$, GERRY DOYLE ${ }^{1}$, CHRIS SKINNER $^{2, *}$ and VINCENZO ANDRETTA ${ }^{3}$}

1 Armagh Observatory, Armagh, N. Ireland

${ }^{2}$ Space Telescope Science Institute, Baltimore MD, U.S.A.

${ }^{3}$ Laboratory for Astronomy and Solar Physics

NASA/Goddard Space Flight Center, Greenbelt MD, U.S.A.

* Deceased 1997 October 21

It is now well-known that many carbon-rich stars - especially those with optically thin dust shells - show a large infrared (IR) excess at 60 and $100 \mu \mathrm{m}$. It is common opinion that such a phenomenon can be explained by assuming that the star is surrounded by a cool detached dust shell, placed far away from it. However, there is no agreement in the literature about the chemical composition or typical size of such detached shells, or their distance from the star. Here we present a set of coeval broadband photometric and spectrophotometric measurements for a sample of carbon stars which show large flux excess in the far-IR. We also present the preliminary results of a spectral analysis carried out considering both oxygen-rich and carbon-rich detached shells:

1. An inner shell composed of amorphous carbon and silicon carbide is required to account for a feature seen in the mid-IR spectrum at low resolution.

2. The inner radius of the detached shell is a few hundred stellar radii (that is, $<0.01 \mathrm{pc}$ ).

3. The far-IR flux excess can be explained by assuming either a detached C-rich shell and an O-rich one; however, with O-rich grains, the dustto-gas ratio required to fit the spectral energy distribution would be too high with respect to what one expects from the cosmic abundances of $\mathrm{Mg}, \mathrm{Fe}, \mathrm{Si}$, and $\mathrm{O}$.

We suggest that the stars we have modelled undergo a short-time-scale modulation in the mass-loss rate, and that the observed detached shell, rather than representing a remnant of the star's former O-rich phase, instead represents a past episode of higher mass-loss rate as a C-rich star. 